\title{
Mujeres creadoras en el sistema del arte de Málaga (2008-2020). Resumen del informe de la Asociación Colectiva Observatorio Cultural Feminista
}

\author{
Isabel Garnelo Díez, Carmen Cortés Zaborras, Belén Ruiz Garrido, María Bueno Castellano, Cristina Savage y Carmen Moreno \\ Universidad de Málaga y Asociación Colectiva Observatorio Cultural Feminista \\ isgadiluna@uma.es; ccortes@uma.es; mrg@uma.es; mbuecastellano@gmail.com; crisavage@gmail.com; \\ carmenmoreno.grafica@gmail.com
}

RESUMEN: El presente trabajo consiste en el estudio de la situación de las mujeres artistas en el sistema del arte de la ciudad de Málaga. La investigación se ha llevado a cabo mediante el recuento de exposiciones organizadas por diferentes instituciones tipificadas como públicas, privadas o independientes y el subecosistema expositivo de la ciudad. El trabajo ha sido realizado por la comisión de investigación de la asociación malagueña Colectiva Observatorio Cultural Feminista, cuyas miembras son a su vez agentes culturales y académicas de la Universidad.

PALABRAS CLAVE: Observatorio cultural; Mujeres en el sistema del arte; Málaga; Perspectiva de género.

\section{Female Artists in the Malaga Art System (2008-2020). Summary of the Report of the Association Colectiva Feminist Cultural Observatory}

ABSTRACT: The present work stems from the study of the situation of the female artists in the art system of the city of Malaga. The research has been carried out by computing the artists' participation at the exhibitions set up in different institutions classified as public, private or independent and the exhibition sub-ecosystem of the city. The work has been conducted by the research commission of the Malaga Association Colectiva Feminist Cultural Observatory, whose members are cultural agents and Málaga University scholars.

KEYWORDS: Cultural Observatory; Women in the Art System; Málaga; Gender Perspective.

\section{Introducción}

Dado que las instituciones públicas son el reflejo de la sociedad en la que desarrollan su trabajo, los museos y centros de arte merecen nuestra atención, porque pueden arrojar luz sobre la posición de las mujeres artistas y la consideración que reciben por parte de las instituciones que también las representan. El observatorio de Colectiva surge, pues, de la necesidad de interrogarnos sobre la situación que viven las artistas que, por otra parte, como se puede fácilmente constatar mediante los informes publicados por MAV y otros colectivos de mujeres artistas, se da en la mayoría de los sistemas del arte de nuestro país y fuera de él. Con este informe queremos dar a conocer la participación de las mujeres en las actividades expositivas organizadas por los centros de arte, salas de exposiciones temporales de los museos, colecciones e instituciones públicas y privadas en Málaga. En este recuento, que empezó en el año 2018, nos propusimos analizar los datos que podrían describir esta situación durante el periodo 2008-2020, para lo que se realizó el recuento y caracterización de las exposiciones individuales y colectivas desarrolladas dentro de las instituciones. Este marco puede verse alterado por el hecho

Cómo citar este artículo: GARNELO DíEZ, Isabel, CORTÉS ZABORRAS, Carmen, RUIZ GARRIDO, Belén, BUENO CASTELLANO, María, SAVAGE, Cristina y MORENO, Carmen, «Mujeres creadoras en el sistema del arte de Málaga (2008-2020). Resumen del informe de la Asociación Colectiva Observatorio Cultural Feminista", Boletín de Arte-UMA, n. ${ }^{\circ}$ 42, Departamento de Historia del Arte, Universidad de Málaga, 2021, pp. 41-48, ISSN: 0211-8483, e-ISSN: 2695-415X, DOI: http://dx.doi.org/10.24310/ BoLArte.2021.vi42.12777 
de que algunas de las instituciones analizadas comenzaron su actividad en años posteriores al 2008. Este año se tomó como punto de partida porque entendemos que, aunque la Ley de lgualdad se aprobó el año anterior, los trámites para su puesta en marcha no se iniciaron hasta el año siguiente. A ello se añade que, en este contexto, la creación en el año 2008 de la Facultad de Bellas Artes en Málaga supuso un hito de gran importancia.

Desde el año 2020, coincidiendo con el confinamiento y las restricciones de movimiento motivadas por la pandemia del COVID19, han surgido en el territorio nacional muchas propuestas desde asociaciones de mujeres y artistas visuales que, de forma colaborativa, ofrecen ideas para mejorar la situación de desigualdad entre artistas mujeres y hombres, constatada en los museos y centros de arte, así como en las salas institucionales y galerías privadas. Dos son las herramientas que se están utilizando en estos momentos en el contexto nacional: la app para el Autodiagnóstico en igualdad y género para museos y centros de arte, desarrollada por MAV, un proyecto que coordina la investigadora Marian L. Fernández-Cao, y el Portal de Igualdad en museos, desarrollado por la artista Mau Monleón. En nuestro contexto local trabajamos para difundirlas, desde la asociación Colectiva y desde las instituciones culturales en las que trabajan algunas de las socias.

En cuanto a la ciudad de Málaga, en la Universidad se han creado en 2020 tanto el IGIUMA, Instituto Universitario de Investigación de Género e Igualdad, como la RIAF, Red de Investigación en Arte y Feminismos. En el tejido social y cultural de Málaga capital se han creado, además de nuestra asociación Colectiva, otras asociaciones: MAGA, de Galerías de Arte profesionales; FAMA, de Fotógrafas Artistas de Málaga; ICC, Plataforma Sectorial de Industrias Culturales de Málaga. Por citar únicamente las relativas a las artes visuales, porque también se han creado otras asociaciones de profesionales de diferentes ámbitos creativos.

En relación con el estudio sobre la igualdad en la participación de mujeres y hombres dentro de las instituciones culturales de la ciudad y la provincia, cabe destacar el informe «Aportaciones al conocimiento de la presencia de las mujeres en el ecosistema expositivo de Andalucía (20082018)», realizado por un equipo de la Universidad de Málaga dirigido por la catedrática Nuria Rodríguez Ortega, publicado en el año $2020^{1}$.
El estudio que hacemos la asociación malagueña Colectiva Observatorio Cultural Feminista se centra en las instituciones públicas y privadas que trabajan en el sector de las artes visuales en Málaga y su provincia. El trabajo se ha hecho gracias a la dedicación desinteresada de las socias de Colectiva, sin ayudas económicas ni subvenciones de ningún tipo. Hemos contado con la ayuda de los y las responsables de las instituciones y galerías observadas, quienes, a petición nuestra, con un grado mayor o menor de colaboración, nos enviaron o facilitaron el acceso a sus programaciones anuales y a los datos necesarios para esta investigación, en un momento en el que el acceso físico a los archivos era imposible. Queremos, pues, agradecerles su colaboración. Otras fuentes importantes de datos han sido los sitios web institucionales, especialmente las páginas de prensa, los catálogos de las exposiciones y los medios de comunicación online.

La intención de este observatorio es conocer la situación de las artistas dentro del sistema del arte de la ciudad: galerías privadas, instituciones públicas, espacios independientes, así como salas de exposiciones en centros educativos públicos. El proceso ha consistido en tomar nota de las exposiciones que cada centro, museo, galería o espacio independiente ha realizado desde el año 2008 -o desde su creación, si inició su actividad posteriormente a esa fecha-, hasta el año 2020, para hacer el recuento de las exposiciones en las que participaron mujeres (si eran colectivas) y de aquellas otras que se dedicaron al trabajo individual de un/ una artista determinado/da, en el caso de las individuales.

No obstante, nuestro estudio no es meramente cuantitativo, es decir, números y porcentajes en crudo, ya que por sí solos no ofrecen alternativas a la situación dada. Hemos creído fundamental conocer los espacios que observamos, las salas y sus condiciones de tamaño, acceso, visibilidad, etcétera; así como las condiciones de publicidad y difusión de las exposiciones en el caso de las y los artistas: catálogos, críticas en medios especializados, notas de prensa, artículos o secciones de cultura en medios de comunicación. Saber si existen diferencias y, de haberlas, analizarlas y obtener las respuestas que nos permitan situarnos en el paisaje de nuestras instituciones culturales y de sus prácticas, conocer los motivos, plantear alternativas, instar al cumplimiento de las normas de buenas prácticas en cultura y a la aplicación de la Ley de Igualdad. Este trabajo, de mayor calado, 
seguirá su curso. Por el momento, este informe se ocupará de la difusión de las cifras y porcentajes, con las alusiones relativas a ciertas diferencias espaciales en determinadas instituciones locales que, por su arquitectura y gestión del espacio, dan lugar a diferencias notables que pasan desapercibidas para quien solamente accede a la información de las exposiciones en los medios de comunicación, pero no ha tenido la oportunidad de visitar las exposiciones in situ. Los resultados obtenidos no difieren demasiado de los ya conocidos y publicados desde otras ciudades e instituciones feministas, tras hacer este mismo estudio en sus localidades o en los ámbitos autonómico y nacional. Todos ellos han servido de inspiración y guía metodológica en el desarrollo del nuestro. Nos referimos especialmente a los informes MAV \#5 (1999-2009), \#12 (2010-2013) y \#19 (2014-2019)² sobre la autoría de exposiciones individuales en museos y centros de arte en España. El informe Arte + Mulleres Creadoras Galegas, realizado por la Sección de Creación y Artes Visuales Contemporáneas del Consello de Cultura Galega, publicado en 2015, bajo la supervisión editorial de María Luisa Sobrino Manzanares ${ }^{3}$. También destacamos, en lo que se refiere al recuento de exposiciones atendiendo a la diferencia entre hombres y mujeres en centros de arte, ferias y museos, las prospecciones realizadas por la artista malagueña Cristina Savage, en las instituciones de Sevilla, Málaga y su provincia, como parte de la investigación previa a sus performances, realizadas en los mismos centros culturales observados, en las que critica la situación de desigualdad de trato entre artistas constatada en ellos.

Al distanciamiento de las instituciones culturales del contexto local, de las redes de creación interior, que son tan importantes, se suma la falta de equidad entre hombres y mujeres en las exposiciones, la adquisición de obras en las colecciones y la participación en actividades programadas por los museos y centros de arte. Aunque esta situación ha mejorado después de las reivindicaciones de las mujeres acontecidas internacionalmente durante el año 2018, aún queda mucho por hacer.

\section{Instituciones públicas y de financiación mixta}

Las instituciones públicas de Málaga capital son peculiares. Difieren bastante de las instituciones de estas características en otras capitales. La razón estriba en un modelo de museo o exhibición de colecciones franquiciado, de financiación pública o público-privada, y en un centro de arte contemporáneo que se gestiona mediante financiación externa privada.

En otro orden de cosas, hacer una búsqueda de las actividades expositivas en las instituciones públicas de Málaga supone entrar en un periplo por los sitios web de diferentes instituciones. Ninguno de los sitios web del Ayuntamiento de Málaga o de la Junta de Andalucía recoge de forma clara, continuada y fiable las actividades desarrolladas en las diferentes sedes de las instituciones culturales que hay en la ciudad. Tampoco en el sitio web de la entidad concreta, cuando existe, hay un apartado específico de exposiciones, con un histórico de las realizadas hasta la fecha actual. Excepciones ejemplares son la del Centro de Arte Contemporáneo de Málaga o la del Museo Picasso Málaga. En este sentido, sorprende la dependencia de las instituciones culturales públicas, ya sean del gobierno de la región autónoma o del Ayuntamiento de la ciudad, a la hora de poder disponer de su propio sitio web, lo que entorpece la labor de difusión pública, transparencia e identidad cultural de las mismas.

\section{INSTITUCIONES PúblicAs \\ Ayuntamiento de Málaga}

- Centre Pompidou Málaga

- Centro de Arte Contemporáneo

- Museo del Patrimonio Municipal

- Fundación Picasso Museo Casa Natal

- Sala de exposiciones Moreno Villa

- Salas Mingorance del Archivo Municipal

Diputación Provincial de Málaga

- La Térmica. Centro de Creación Contemporánea

- Centro Cultural Provincial María Victoria Atencia Junta de Andalucía

- Museo de Málaga

- Museo Picasso Málaga

- El Palmeral Espacio Iniciarte

- Escuela de Arte San Telmo

Universidad de Málaga

- Salas: Rectorado, Muralla y Espacio Cero UMA

- Galería Central Facultad de Ciencias de la Comunicación UMA

- Galería Facultad de Bellas Artes de la UMA 


\section{Instituciones privadas e independientes}

Las instituciones privadas de Málaga capital consideradas en este estudio son las que, además de sus actividades económicas o de cualquier otra índole posible, realizan actividades expositivas y tienen para ello centros o salas de exposición en sus sedes u otros locales de la ciudad. Este sería el caso de la Fundación Unicaja.

Contemplamos aquí tanto las galerías de arte tradicionales como ciertos espacios independientes, a menudo autofinanciados, que han crecido exponencialmente en los últimos años. Estos espacios suelen estar dirigidos por artistas y resulta interesante conocer las formas de organización, trabajo colaborativo con los/las creadoras y alcance de su trabajo para los y las artistas que integran sus organizaciones. Estos espacios, que cuentan con un local en el centro de la ciudad de Málaga, realizan exposiciones, pero también residencias de creadores/as, talleres y, en contadas ocasiones, comisariado para instituciones públicas. También han dado lugar, como es el caso de La Casa Amarilla o el Taller-Galería Gravura, a iniciativas que han ampliado el conocimiento de las actividades y el trabajo de los/las artistas que se mueven en la órbita de estos espacios. No solo en el campo de la creación visual, sino también en el del turismo o las artes escénicas. Siempre desde una perspectiva de puesta en valor del trabajo de los creadores/as y buscando su inserción y profesionalización dentro del sector cultural local. En este sentido, también las galerías de arte han creado recientemente la asociación MAGA con el objetivo de mejorar su situación en relación con las instituciones públicas en la promoción de sus artistas y la obtención de reconocimiento y financiación pública.

Es reseñable que, como ocurre en las instituciones públicas, la participación de las mujeres se incrementa cuando se trata de exposiciones colectivas.

\section{INSTITUCIONES PRIVADAS O INDEPENDIENTES}

- Museo Carmen Thyssen Málaga

- Ateneo de Málaga

- Fundación Unicaja

- La Casa Amarilla

- Galería Taller Gravura

- Galería El Estudio de Ignacio del Río

- Yolanda Ochando. Obra Gráfica
- Galería Isabel Hurley

- Galería Javier Marín

\section{Espacios del subecosistema expositivo ${ }^{4}$}

El tercer bloque de espacios considerados en este informe recoge los pertenecientes al denominado subecosistema expositivo de la ciudad de Málaga. Son espacios que no son galerías estrictamente hablando, ni su actividad comercial o profesional se restringe a las artes visuales. En este grupo podemos encontrar desde bares, librerías o estudios de artistas y talleres artesanales dirigidos por artistas o artesanas, hasta modelos de galería y comisariado muy profesionales, que desarrollan su labor entre las redes sociales y su propia vivienda. Este último caso sería el de Casa Sostoa (2013), de la que fue un precedente La Casa Amarilla en sus inicios, inaugurada en 2008, o La Casa de Blas, inaugurada el año 2011. Otro precedente, hoy extinto, pero que fue de gran importancia para las nuevas formas de gestión que se han ido desarrollando en la ciudad, lo constituye el Espacio Cienfuegos (2012), abierto por un grupo de artistas universitarios multidisciplinar. Cienfuegos tenía el valor de trabajar bajo presupuestos críticos con el, entonces en pleno desarrollo, contexto de gentrificación/turistización social y cultural de Málaga, mediante una continuada puesta en acción de alternativas de vida y lectura del entorno, para los/las artistas y para los/las habitantes de la ciudad.

\section{ESPACIOS DEL SUBECOSISTEMA EXPOSITIVO}

- Casa Sostoa

- Antinoo Art Gallery

- El Retorno de Lilith

- Galería Valmar

- Isla Negra. Librería Galería

- La Matraca

- La Polivalente

- Mahatma Showroom

- Taita Studio

\section{Resumen de los resultados}

A la luz de los datos obtenidos en el recuento realizado, los resultados sobre la relación de los artistas y las artistas par- 


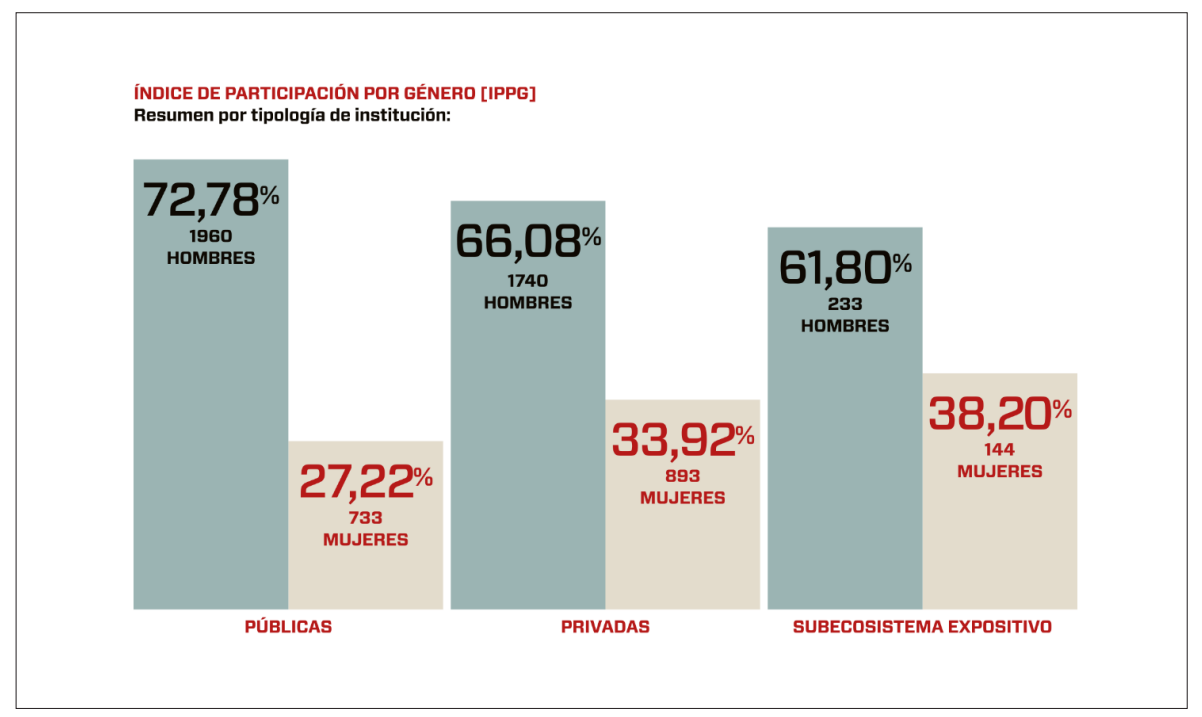

ticipantes en las actividades programadas por museos, centros de arte y galerías están muy lejos de la paridad. Dentro del ecosistema expositivo de la ciudad, las instituciones observadas han sido en total 18 entidades públicas, 7 privadas y 9 espacios englobados dentro de la categoría de subecosistema expositivo. Sin embargo, en relación con el índice de participación por género en exposiciones, jurados, colecciones, etcétera, los números dibujan una situación bastante parecida entre entidades públicas y privadas, con una inclinación de la curva hacia la desigualdad en lo que respecta a las exposiciones/colecciones, por cierto, más pronunciada en el caso de las instituciones públicas.

En estas últimas, el índice general de participación por género desciende negativamente en espacios como las salas de La Sociedad Económica de Amigos del País con un 12,3\%, las de la Universidad de Málaga con un 16,2\%, el Museo de Málaga o el Museo Picasso Málaga, ambos con un 10\% de índice general de participación femenina, y es especialmente dramática en la Fundación Picasso Casa Natal, con un $3,32 \%$.

Todo ello debería apelar a tomar conciencia de la situación para revertir de forma voluntaria las políticas que están provocando esta situación desigual. También es necesario hacer un ejercicio de contemporización, ya que hay instituciones, como la Universidad de Málaga, que lleva programando exposiciones activamente desde el año 2008 y otras, como por ejemplo el Museo Pompidou, llevan abiertos desde el año 2015. Al mismo tiempo, hay que valorar que, mientras en la Universidad ha habido cambios en la dirección de cultura con una periodicidad más corta, en el Museo Picasso Málaga, como en otras instituciones culturales públicas y privadas de las observadas, los cargos suelen tener una duración mucho más larga en el tiempo y abarcan gran parte del periodo de estudio determinado en este informe. Por no hablar de que la Universidad no es un centro cultural, ni una galería de arte, ni un centro de arte contemporáneo.

Pero no creamos que en el resto de las instituciones consideradas la situación mejora. Más bien todo lo contrario. Sobre todo si tenemos en cuenta que la Facultad de Bellas Artes abre sus puertas en Málaga precisamente en 2008, lo cual podría hacer esperar la salida de muchas más artistas profesionales con una mayor formación en el conocimiento de las estrategias de consolidación de su carrera a medio y largo plazo, por no hablar de una alta conciencia feminista. Este año, como ya hemos señalado, supone el comienzo de la andadura de la Ley de Igualdad, que pone el énfasis en la necesidad de cambiar el modo en que se consideran las producciones de las mujeres, además de plantear explícitamente que el desplazamiento y en muchos casos el ocultamiento histórico sufrido por estas es una tara del sistema, no de las artistas. Así, vemos que una institución que podría ser menos sospechosa de machismo, por su contemporaneidad y juventud, como el Centro de Arte Contemporáneo de Málaga, arroja un 20,2\% de participación de mujeres artistas 
en el periodo que va desde el año 2008 al 2020, frente al $79,8 \%$ de sus colegas masculinos. Tampoco el Centre Pompidou, aunque fue inaugurado en el año 2015, parece contar entre sus prioridades la de paliar esta situación, tanto en las propuestas de las exposiciones permanentes como en las de exposiciones temporales. Por lo que se refiere a estas últimas, en los cinco años de actividades desarrolladas, el índice general de participación femenina ha sido del 24,6\% frente al 75,3\% de participación masculina. En las Salas de la Coracha, en el MUPAM, se reproducen estas cifras claramente discriminatorias, con un $27,48 \%$ de participación femenina frente al $72,52 \%$ de la masculina. Como ya se ha apuntado, es importante señalar que la participación femenina se ha dado principalmente en exposiciones colectivas y no, como ocurre con sus colegas masculinos, en exposiciones individuales, en las que es posible mostrar con amplitud una obra y una trayectoria artística y profesional que pueda quedar legitimada por la institución y la crítica.

Podríamos seguir con las demás instituciones públicas y las salas que tienen en diferentes zonas de la ciudad, de mayor o menor tamaño, con agendas y programaciones más o menos coherentes, más o menos erráticas. Si bien es cierto que en algunos de estos casos los números mejoran algo para las mujeres artistas, lo cierto es que la diferencia con sus coetáneos masculinos sigue siendo muy marcada.

Como decíamos, el índice general de participación es un valor que no nos permite describir con exactitud la gravedad de la situación. Podemos verlo si nos detenemos en el ejemplo del Centro de Creación Contemporánea La Térmica. Aquí, este índice general arroja unos porcentajes de participación de mujeres artistas bastante aceptables, de 41,93\% frente al 58,07\% de hombres. Pero si vamos al porcentaje extraído de las exposiciones individuales, esto cambia drásticamente, con una relación del 26,20\% de mujeres artistas frente al 73,80\% de hombres artistas. Lo mismo podría concluirse con respecto al Centro Cultural María Victoria Atencia, también gestionado por la Diputación de Málaga, en el que encontramos el 32\% de artistas mujeres frente al $68 \%$ de artistas varones. Sorprende, en cualquier caso, que estos resultados sean independientes de que la sala, el museo, la galería o el centro de arte, esté dirigido o gestionado por un hombre o por una mujer.

Esta tendencia solo mejora en aquellas salas dependientes de centros educativos, o bien con un carácter inde- pendiente o autofinanciado, cuyo fin no es comercial ni su actividad puede ser considerada profesional en un sentido estricto. Aquí podríamos comentar la situación que dibujan espacios o salas como la Galería Central, de la Facultad de Ciencias de la Comunicación, con una relación de participación de hombres y mujeres de 44,80\% y 55,20\% respectivamente, la única en la que se invierte la tendencia habitual; la sala de la Facultad de Bellas Artes, que si bien abrió mucho más tarde, arroja unos índices de 48,28\% de artistas mujeres frente a $51,72 \%$ de varones, cifras que parecen más realistas y acordes con el número de matriculadas y egresadas de esta facultad; la sala de la Escuela de Arte San Telmo, con una relación de 47,5\% frente al 52,5\% respectivamente; o, por último, el Ateneo de Málaga, con una participación femenina del 33,60\% frente al 66,40\% de masculina. Como vemos, en la mayoría de ellas, a pesar de todo, sigue siendo más alta la selección de participación masculina.

En las instituciones privadas malagueñas consideradas, los porcentajes son similares a los observados en las públicas. Tanto el Museo Carmen Thyssen, con una relación de participación femenina y masculina de 15,5\% y $84,5 \%$ respectivamente, o la Fundación Unicaja, con un 33,60\% frente al 66,24\%, cifras estas que son más equilibradas que las de la anterior entidad, pero en las que vemos que aún queda mucho por hacer para mejorar la situación. Por su parte, las galerías de arte privadas mantienen una tónica similar entre ellas. Así, encontramos que galerías con más trayectoria y una actividad profesional consolidada en la ciudad, como Isabel Hurley y Javier Marín, presentan un índice de participación por género mujer-hombre del 40\%-60\% y del 33,9\%-66,07\%, respectivamente. En ambos casos sorprende el que esto sea así, porque el número de mujeres y hombres artistas representados por ambas galerías es equilibrado, de hecho, en el caso de la galería Javier Marín las artistas y los artistas coinciden en número.

Bajan un poco los índices de participación femenina en el caso de Yolanda Ochando Obra Gráfica y El Estudio de Ignacio del Río, que no son comparables debido a la diferencia de tiempo activo de cada una de ellas. Yolanda Ochando abre sus puertas en el año 2004, mientras que El Estudio de Ignacio del Río lo hace en 2012. También se diferencian en el trabajo desempeñado, Yolanda Ochando no realiza estrictamente el trabajo de una galería de arte convencional, la propia directora habla de presentaciones en lugar de expo- 
siciones. Ambos espacios presentan un índice de participación mujer-hombre del 25,45\%-75,55\% y el 26,7\%-73,3\% respectivamente.

Dentro de la categoría de instituciones privadas, se han incluido también la Galería-Taller Gravura y La Casa Amarilla. Al igual que con las dos anteriores, estos espacios también se diferencian enormemente: por la fecha de apertura, pues Gravura abre sus puertas en el año 1980, mientras que La Casa Amarilla lo hace en 2008; por el modelo de empresa que representan, aunque la segunda se ha visto inspirada por la primera. No obstante, en ambas se ha demarcado el tiempo de observación en el periodo 2008-2020, por las razones que ya hemos explicado al principio de este análisis y porque sus trayectorias lo han permitido. Los índices de participación en estos dos casos, mujeres y hombres respectivamente, son del 44,98\%-55,02\% en La Casa Amarilla y del 38,98\%-61,01\% en Gravura. Observamos que las categorías de selección empleadas en estos dos últimos espacios no son tan restrictivas como las aplicadas en las galerías privadas consideradas tradicionales. De hecho, son espacios que trabajan sobre todo con artistas locales. Por este motivo, las actividades que desarrollan La Casa Amarilla y la Galería-Taller Gravura no se limitan únicamente a la exposición y promoción de los/las artistas de la galería, sino que su financiación proviene también de actividades paralelas, como los cursos, los talleres, el comisariado o la gestión cultural externa, más en sintonía con el centro de arte o la institución cultural pública.

Por último, la tercera categoría de espacios observados para la realización de este informe es la que hemos denominado subecosistema expositivo de la ciudad, bajo cuyo paraguas estarían instituciones menos estructuradas, más efímeras o polivalentes que las descritas hasta aquí. Si nos ha interesado incluir estos espacios dentro de nuestro estudio, ha sido precisamente por la diferencia y diversidad que presentan con respecto a los espacios institucionales o institucionalizados a lo largo de los últimos doce años. También, porque muchos de ellos están dirigidos por artistas, como ya ocurría con algunos de los incluidos en la categoría anterior, pero que se han desvinculado de ellos por el tiempo que llevan en activo, muy inferior al de aquellos, por la juventud de sus directores y por las zonas de la ciudad en la que se ubican, a menudo situados en la periferia de la ciudad. Nos interesaba ver si la dirección de estos espacios, los/las artis- tas que los gestionan y las actividades que desarrollan, tanto de difusión del trabajo de otros/as artistas, como de las actividades que les permiten financiarse, suponían un cambio en los porcentajes de participación analizados en nuestro estudio.

La primera de las diferencias con respecto a los espacios institucionales públicos o privados analizados bajo los presupuestos descritos es, desde luego, el tiempo de actividad, ya que estos espacios inician su andadura entre 2013 y 2020. En segundo lugar su carácter, como decíamos, polivalente. Todos ellos tienen otra función principal o secundaria que va desde la librería al bar de copas, pasando por el estudio de artista o arquitecto que se convierte en espacio expositivo, o la casa privada que deviene provisionalmente galería de arte.

Desde el inicio de la pandemia del COVID19, emerge con fuerza un triángulo cuyos vértices son lo local, la red y la persona artista como verdaderos protagonistas de un cambio que empieza por lo individual. Creemos que muchos de estos espacios dirigidos por artistas están trabajando teniendo en cuenta estas tres variables, además de apostar por la artesanía, cerámica y textil principalmente, sin perder de vista el contexto global en su riqueza y pluralidad. Seis de estos espacios están dirigidos por hombres y tres por mujeres, El Retorno de Lilith, la Galería Valmar y Taita Estudio. Los porcentajes de participación en ellos son, considerando a mujeres y hombres en este orden: Casa Sostoa: 34,93-65,07\%; Antinoo Fine Art Gallery: 43,5\%-56,5\%; El Retorno de Lilith: 47,2\%-52,7\%; la Galería Valmar: 16,7\%-83,3\%; Isla Negra Librería Galería: 14,2\%-85,5\%; La Matraca: 42,1\%-57,9\%; La Polivalente: 39,5\%-60,5; Mahatma Show Room: 34,6\%65,4\%; Taita Estudio: 83,34\%-16,66\%.

Como puede apreciarse, la participación de las mujeres artistas es destacada en las programaciones de estas salas. Esto se debe principalmente, en algunas de ellas, a que la mayoría de exposiciones programadas son colectivas, en las que participan muchas más mujeres, con lo cual se produce este incremento que ya apreciamos en las instituciones públicas y privadas analizadas con anterioridad. Esta tendencia en este tipo de espacios se invierte solamente en el caso de Taita Estudio, donde, como vemos, los porcentajes se inclinan, felizmente, a favor de las mujeres artistas. Esta tendencia se mantiene en el caso de las exposiciones individuales, que constituyen la mayor parte de 
la actividad de Taita Estudio (con una relación del 83\%-17\% entre mujeres artistas y hombres artistas).

Hasta aquí hemos querido presentar el paisaje de la situación de las mujeres artistas en el sistema del arte de nuestro entorno local. Si debido a la impresión que los números suelen producir nos parece que la situación no es buena, es porque no lo es. Queremos que sirva a todas las instituciones públicas y privadas, para que estos resultados lleven a una profunda reflexión y sean, a su vez, un incentivo para trabajar en pos del máximo equilibrio en la participación de las mujeres artistas en el sistema del arte de Málaga. Esperamos sinceramente que este Observatorio pueda servir para tener una visión lo más realista posible y que permita tomar conciencia de la situación descrita, de tal forma que puedan aplicarse las políticas de gestión necesarias para revertir esta situación.

\section{Notas}

1 «Aportaciones al conocimiento de la presencia de las mujeres en el ecosistema expositivo de Andalucía (2008-2018)». Rodríguez-Ortega, Nuria, Cruces Rodríguez, Antonio y Pino Díaz, José, "Mujeres en el ecosistema expositivo andaluz (2008-20018) (I). Artistas y comisarias». Informes Andalex \#1, 18 de mayo de 2020. En: <https://andalexproject.es/wp content/uploads/2020/06/Sexos_02_final_publicado.pdf> (fecha de consulta: 30-12-2020).

2 <https://mav.org.es/category/documentacion/informes/> (fecha de consulta: 30-12-2020).

3 <http://consellodacultura.gal/mediateca/extras/CCG_2015_Arte-Mulleres-Creadoras-galegas.pdf> (fecha de consulta: 30-12-2020).

4 «Subecosistema expositivo» es la denominación dada por el equipo de trabajo del proyecto Andalex Exhibition Project (<https://andalexproject.es/ documentaci\%C3\%B3n/aportaciones-al-conocimiento-de-la-presencia-de-las-mujeres-en-el-ecosistema-expositivo-de-andalucia-2008-2018/>) a los espacios que desarrollan actividades expositivas al margen de los circuitos institucionales o privados tradicionales y que, generalmente, están gestionados por artistas o jóvenes emprendedores. Como en la actualidad algunos de los espacios que según este estudio entrarían en esta categoría están perfectamente integrados en la vida cultural de la ciudad y constituyen tradicionalmente un espacio consolidado de exposición y desarrollo profesional reconocido, creemos necesario en este estudio considerar su definición, para incluir en esta categoría los espacios que efectivamente son dirigidos por artistas o por libreros u otros profesionales, pero que llevan a cabo un trabajo de exposición que es interesante o del que nos parece necesario dejar constancia. 